\title{
Sum of Areas
}

National Cancer Institute

\section{Source}

National Cancer Institute. Sum of Areas. NCI Thesaurus. Code C96630.

A calculation of the aggregated area values. 